\title{
A Multi-Dimensional Super-Orthogonal Modulation Alternative to M-QAM WCDMA for Next Generation Wireless Applications
}

\author{
Louis P. Linde, Senior Member, IEEE and Jacobus D. Vlok
}

\begin{abstract}
This paper presents bit-error-rate (BER) performance results of an upwards-expandable Multidimensional super-orthogonal modulation alternative to M-ary quadrature amplitude modulation wideband codedivision-multiple-access (M-QAM WCDMA), suitable for application in next generation WLAN and wireless cellular systems. The unique combination of a $M=4$-dimensional WCDMA modem configuration and super-orthogonal families of quasi-complementary root-of-unity filtered (RUF) near constant-envelope generalized-chirp-like complex spreading sequences (SO-CE-GCL-CSS), renders a spectrally and power efficient output signal with a data throughput rate equivalent to that of $M=16$-ary quadrature amplitude modulated (16-QAM) WCDMA, but with the BER performance equivalent to that of BPSK/QPSK in both additive white Gaussian noise (AWGN) and fading multipath channel scenarios, given identical spreading factors and bandwidths.
\end{abstract}

Index Terms - Complex Spreading Sequences (CSS), Multi-Dimensional (MD) Modulation, Multi-Layered Modulation (MLM), Super-Orthogonal (SO) Quasicomplementary Sequences (QCS), Root-of-Unity Filtering (RUF), WCDMA.

\section{INTRODUCTION}

$\mathrm{V}$ ARIANTS of multi-carrier orthogonal frequency division multiplexing $\left(M_{c}\right.$-OFDM, including $M_{c}$-CDMA) have been successfully introduced in the 802.11a and $\mathrm{g}$ (WiFi) WLAN standards, as well as in broadband wireless access standards such as 802.16-2004 (WiMax). The success of these spectrally efficient modulation schemes have spawned the idea of extending their application to next generation single carrier wireless cellular systems, that have traditionally been based on $2.5 \mathrm{G}$ and $3 \mathrm{G}$ WCDMA modulation standards.

The spectral efficiency and data throughput advantages that

Manuscript received March 3, 2007. This work was supported in part by the South African NRF under a four-year Grant, entitled: "IP-based Rural Communication System", GUN:2053415, dated Jan. 2003.

L. P. Linde is with the Department of Electrical, Electronic and Computer Engineering, University of Pretoria, Pretoria, 0002 South Africa. (Phone: +27 12420 2168, fax: +27 12362 5000, e-mail: 1linde@ postino.up.ac.za).

J. D. Vlok, was with the University of Pretoria, Pretoria 0002, South Africa). (Cell: +2782484 2607).
$M_{c}$-OFDM-related modulation schemes exhibit over existing $3 \mathrm{G}$ single-carrier UMTS and cdma-2000 modulation standards, are however hampered by the weak power efficiency of the former schemes, as manifested by their high peak-toaverage power ratios (PAPR), as well as their sensitivity to mobility and fast fading. The high OFDM PAPR requires power amplifier (PA) back-offs of typically 6 to $9 \mathrm{dBs}$ to prevent signal amplitude saturation and corresponding spectral regrowth [1],[2] resulting in limited communication range due to inefficient utilization of available handset battery power. The OFDM performance and power inefficiency is further jeopardized by the use of M-ary quadrature amplitude modulation (M-QAM) of individual orthogonal subcarriers in an effort to achieve improved spectral efficiency [3]. The performance degradation due to the use of these modulation schemes cannot be neutralized or improved without the inclusion of sophisticated forward error correction (FEC) and/or PAPR control coding mechanisms. This paper proposes an alternative low-complexity upwards-expandable generic single carrier $\left(M_{c}=1\right)$ QS-4D-SO-WCDMA modulation scheme with the potential to overcome the PAPR problems (even with $M_{D} \geq 4$ dimensions), while achieving significant BER performance gains which are QPSK-like, compared to single carrier and $M_{c}$-OFDM and $M_{c}$-CDMA $M$-QAM modulation standards [4]. The BER performances of the latter systems degrade significantly compared to QPSK when the number of signal levels (or symbols) increases beyond $M_{L}=4$ (or $M=16$ ).

The outline of the rest of the paper is as follows: The transmitter and receiver architectures of the new quasisynchronous multi-code multi-dimensional super-orthogonal WCDMA (QS-MC-MD-SO-WCDMA) modulation technique are described in section II. This is followed by a theoretical analysis of the BER performance of the proposed system in both AWGN and multipath Rician/Rayleigh fading channel conditions in Section III, with and without the presence of a complex RAKE multipath combiner in the receiver. A conclusion of the primary findings of this research project is finally given in Section IV, followed by a list of references cited during this project. 


\section{QUASI-SYNCHRONOUS MULTI-CODE MULTI- DIMENSIONAL SUPER-ORTHOGONAL WCDMA}

The new QS-4D-SO-WCDMA modem building block, depicted in Fig. 1, and the use of unique extended families of root-of-unity-filtered (RUF) [5] constant-envelope generalized chirp-like [6] complex spreading sequences (CE-GCL-CSS), exhibiting zero periodic cross-correlation (ZCC) values for all relative time shifts $\tau$ [7]-[9] (henceforth referred to as superorthogonal (SO) sequences), together render a novel minimum (Nyquist) bandwidth near constant envelope (or instantaneous power) output signal, while simultaneously facilitating multiuser-interference (MUI) free detection, even in the presence of rapidly varying multipath fading channel conditions [10]. This enables the use of significantly simplified correlationtype receiver structures without the need for complex MUIcancellation mechanisms or sophisticated PAPR control measures normally required for $M_{c}$-OFDM-based systems. Whereas OFDMA schemes achieves multi-dimensionality and improved spectral efficiency (in bits/s/Hz) via a fixed set of $M_{c}$ mutually orthogonal quadrature subcarriers and $M$-QAM modulation per subcarrier, single carrier QS-4D-SO-WCDMA achieves multi-dimensionality and improved spectral efficiency through a multi-code MLM modulation approach, comprising the superposition of combinations of the point-bypoint orthogonal real and imaginary parts ${ }^{1}$ of sets of RUF SOCE-GCL-CSS of length $L$ chips, and the quadrature components of a carrier, which are individually binary phase and frequency, and optionally, also code-position-modulated (CPM), by independent sets of four (or more) parallel binary symbol streams per CSS.

The proposed new spectrally and power efficient families of SO-CE-GCL-CSS are derived from the zero-cross-correlation (ZCC) sequences proposed in [8], by applying a non-linear root-of-unity-filtering technique presented in [5]. In fact, by applying the RUF-process to any GCL family of sequences, a novel near constant-envelope (CE) minimum (Nyquist) bandwidth family is produced which retains the unique correlation properties of the family it was derived from [8]. The use of the new families of SO-CE-GCL-CSS requires a synchronous or at least a quasi-synchronous communication scenario in both the down- and up-links of the wireless access system. The accuracy of the synchronisation system and the maximum delay spread that can be tolerated are primarily functions of the cell size and the geometry of the environment that affects the RF propagation characteristics. The maximum allowable delay or multipath spread that can be accommodated in an operational environment is determined by the char-acteristics of the periodic auto-correlation function (PACF) of the proposed new family of spreading sequences [7]-[9] and is typically limited to a few (say 10 to 20) chips of the spreading sequences employed.

\footnotetext{
${ }^{1}$ The real and imaginary components of each RUF CE-GCL-CSS are in fact Hilbert transforms of one-another
}

\section{A. MD-SO-WCDMA Modem Transmitter Description}

The generation of the 4D-SO-WCDMA output signal $s_{l}(t)$ may be best described as the sum of sets of four \pm 1 Volt NRZ binary symbol streams $\left\{d_{l, m}(t)\right\} ; m=1,2,3,4$ in parallel, each phase modulating the $l^{\text {th }}$ orthogonal base $\left\{\Psi_{l, m}(t)\right\}, m=1,2,3,4$; $l=1,2 \cdots M_{F}$, where the individual $\Psi_{l, m}(t)$ is formed by combinations of products of the perfectly orthogonal real and imaginary parts of the $t^{\text {th }} \operatorname{CSS}, \operatorname{CSS}_{l}(t)=C_{l, 1}(t)+j . C_{l, 2}(t)$ (denoted as $C_{1}$ and $C_{2}$ in the $4 \mathrm{D}$ systems of Figures 1 and 2, respectively $^{2}$ ) from a family of $M_{F}$ SO-CE-GCL-CSS, and the in-phase and quadrature components of a quadrature carrier, $\left\{\cos \left(\omega_{c} t\right),-\sin \left(\omega_{c} t\right)\right\}$. Let $t^{\prime}=\left(t-n T_{s}\right), T_{s}$ denote the symbol period and $\sqrt{T_{s}} / 2$ be a normalization factor. Then:

$$
\begin{array}{r}
s_{l}(t)=\sqrt{T_{s}} / 2 \sum_{n=-\infty}^{\infty}\left[\left\{d_{l, 1}\left(t^{\prime}\right) C_{l, 1}\left(t^{\prime}\right)+d_{l, 2}\left(t^{\prime}\right) C_{l, 2}\left(t^{\prime}\right)\right\} \cos \left(\omega_{c} t\right)\right. \\
\left.-\left\{d_{l, 3}\left(t^{\prime}\right) C_{l, 1}\left(t^{\prime}\right)+d_{l, 4}\left(t^{\prime}\right) C_{l, 2}\left(t^{\prime}\right)\right\} \sin \left(\omega_{c} t\right)\right] \\
=\sqrt{T_{s}} / 2 \sum_{n=-\infty}^{\infty}\left[\sum_{m=1}^{M_{D}=4} d_{l, m}\left(t^{\prime}\right) \psi_{l, m}\left(t^{\prime}\right)\right]
\end{array}
$$

The $\left\{\Psi_{l, m}(t)\right\}, i=1,2,3,4$; where index $l$ denotes one CSS from a family of $M_{F}$ complex spreading sequences of length $L$ chips, form an orthonormal base over each symbol period $T_{s}=4 T_{b}$, spanning the $M_{D}=4$-dimensional Euclidian space, with $T_{b}$ the bit period. The data throughput rate of one basic 4D-SO-WCDMA building block is twice that of a QPSK DSSS modulation system, yielding a spectral efficiency of $4 / \mathrm{L}$ $[\mathrm{b} / \mathrm{s} / \mathrm{Hz}]$, i.e., equal to that of $M=16-\mathrm{QAM}$, but with the BER performance of BPSK/QPSK, if identical spreading sequence lengths $L$ and equal spreading bandwidths $B_{s}$ are assumed. It is straightforward to show that the 4D-SO-WCDMA modem transmitter building block depicted in Fig. 1 may be extended to more dimensions $\left(M_{D}>4\right)$ by adding more superorthogonal 4D-blocks in parallel, each using only one additional CSS from a family of $M_{F}$ CE-GCL-CSS for every quadruple increase in the number of dimensions, to yield the composite MD-SO-WCDMA output signal $s(t)=\sum_{l=1}^{M_{F}} s_{l}(t)$. This is achieved while maintaining a QPSK-like BER performance, i.e., with significant SNR advantages compared to $M$-QAM modulated single carrier and $M_{C^{-}}$WCDMA modulation schemes for a given BER-performance, as will be illustrated in section III A (with specific reference to Table 1).

\section{B. MD-SO-WCDMA Modem Receiver}

A block diagram of the corresponding basic QS-4D-SOWCDMA matched filter (integrate-and dump) receiver is depicted in Fig. 2.

\footnotetext{
${ }^{2}$ Note that $C_{1}$ and $C_{2}$ in Figures 1 and 2 either depict the real or imaginary parts, or the sums and differences of the real and imaginary components of one CSS, respectively - see [4] and [11] for more details.
} 


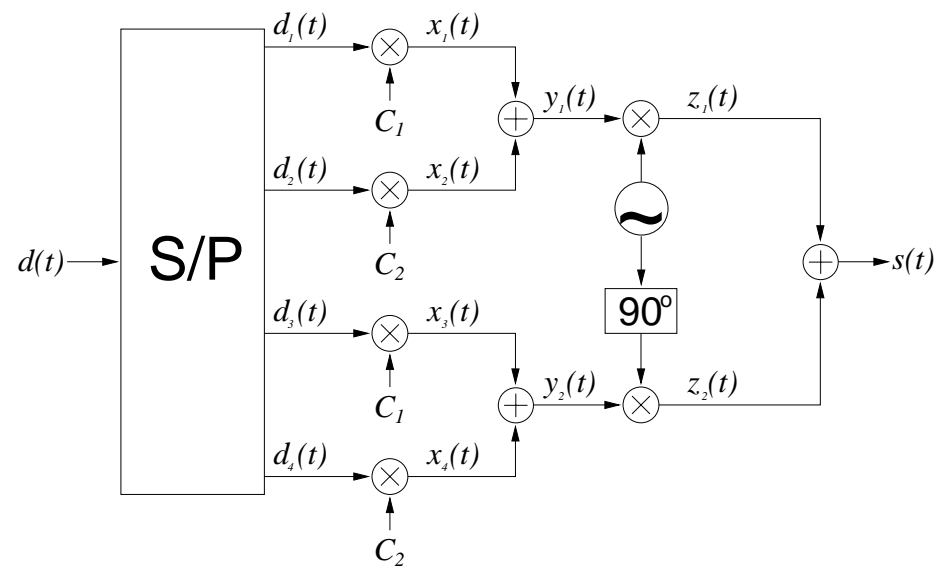

Fig. 1 Uncoded basic Quasi-Synchronous 4-Dimensional SuperOrthogonal Wideband Code Division Multiple Access (QS-4D-SOWCDMA) modem transmitter building block.

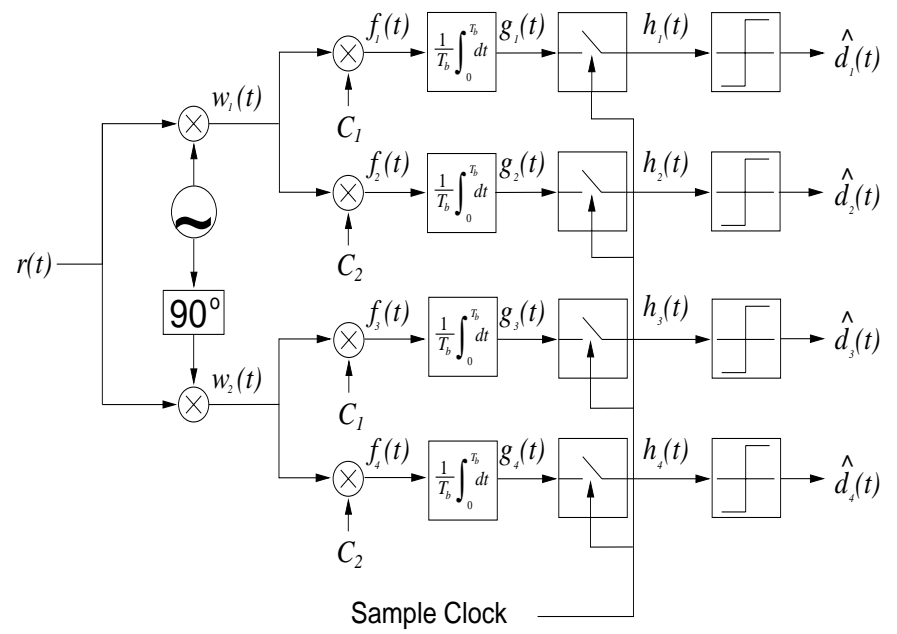

Fig. 2 Correlation-type QS-4D-SO-WCDMA receiver block diagram.

The received signal, $r(t)$, is split into two branches, quadrature carrier demodulated and low-pass filtered to remove double frequency carrier components. The resultant demodulated in-phase $w_{l}(t)$ and quadrature-phase $w_{2}(t)$ signals are again split into two branches, respectively, before being complex despreaded by the $l^{\text {th }}$ CSS, say $\operatorname{CSS}_{l}(t)=C_{l, 1}(t)+j C_{l, 2}(t)$, from a family of $M_{F}$ SO-CE-GCLCSSs. The outputs $\left\{f_{m}(t)\right\}, m=1,2,3,4$ from the complex despreader are then integrate-\&-dumped (I\&D), to yield outputs $\left\{g_{m}(t)\right\}, m=1,2,3,4$, which are then optimally sampled to produce the decision variables $\left\{h_{m}(t)\right\}, m=1,2,3,4$. Finally, estimates of the original four serial-to-parallel converted symbol streams $\left\{d_{m}(t)\right\}, m=1,2,3,4$, depicted in Fig. 1, are obtained through binary decision devices with thresholds set to zero, to produce the final symbol estimates, $\left\{\hat{d}_{m}(t)\right\}$, $m=1,2,3,4$. Note that the combination of the quadrature demodulation and complex despreading is equivalent to despreading with a SO base $\left\{\Psi_{l, m}(t)\right\}, m=1,2,3,4$, associated with the $t^{\text {th }}$ CSS from the family of $M_{F}$ CSS, i.e., the inverse of the complex spreading process depicted in Fig. 1. Also note the simplicity of the basic QS-4D-SO-WCDMA receiver, and particularly the absence of a complex MUI-cancellation device as a result of the use of families of SO-CE-GCL-CSS in the spreading process. More attention may therefore be focused on synchronization and the incorporation of a complex RAKE processor to exploit any form of inherent multipath diversity in the received WCDMA signal $r(t)$. Details of the latter processor may be found in [18].

Perfect carrier and symbol timing recovery is assumed in this paper - details of the high-performance carrier synchronization and the complex code-lock-loop (CCLL) have been described in detail in [11] and will not be elaborated on here.

\section{QS MULTI-CODE MD-SO-WCDMA MODEM PERFORMANCE ANALYSIS}

\section{A. BER Performance in AWGN}

It will now be demonstrated that the uncoded AWGN BER performance of the proposed MD-SO-WCDMA configuration remains BPSK/QPSK-like with increasing dimensions $M_{D}$, by virtue of the perfect periodic cross-correlation (PCC) properties amongst the spreading sequences of the family proposed in [8].

Let $M_{L}$ denote the number of symbol levels per dimension, $M_{D}$. In the following discussion, it is also assumed that both the spreading bandwidth and the spreading sequence length $L$ are fixed. The WCDMA processing gain (PG) is defined as the ratio of the chip rate to the symbol rate. Then, the total number of symbols $M$ and the corresponding number of bits per symbol $K$ is given by

$$
M=\left(M_{L}\right)^{M_{D}}=2^{K} \quad[\text { symbols }]
$$

The subsequent analysis is based on the fact that, in general, the MD-SO-WCDMA signal constellation is a $M_{D^{-}}$ dimensional hypercube centered on the origin of the signal space spanned by an orthonormal base, such as the one defined in (1) [12]. In fact, with $M_{D}=4$ and $M_{L}=2$, the $M=2^{M_{D}}=16$ symbols are situated on the vertices of an $M_{D}=4$-dimensional hypercube, where the number of bits per symbol equals $K=M_{D}=4$. The latter relationship is a characteristic of an $M_{D}$-dimensional hypercube. Note that each dimension of the MD-SO-WCDMA signal space only carries one bit of information, i.e., according to (1), the MD-SOWCDMA modulator only performs binary $\left(M_{L}=2\right)$ modulation of GCL CSS per dimension. Furthermore, the coordinates of nearest-neighbour symbols only differ in one bit position, i.e., the signal constellation exhibits a natural Gray mapping of bits onto symbols.

It is well known from [13] that the correlation $\rho_{r}$ amongst all adjacent pairs of symbols in the $M_{D}$-dimensional hypercube signal space is given by: 


$$
\rho_{r}=\frac{M_{D}-2}{M_{D}}
$$

Furthermore, using (3), the Euclidean distance between nearest-neighbour symbols can be shown to be:

$$
d=\sqrt{2 E_{s}\left(1-\rho_{r}\right)}=\sqrt{\frac{4 E_{s}}{M_{D}}}
$$

Since the symbol energy equals $E_{s}=M_{D} \cdot E_{b}$, with $E_{b}$ the energy per bit, $d$ simplifies to $\sqrt{4 E_{b}}$, and the symbol error rate (SER) of the MD-SO-WCDMA modulation scheme in AWGN is approximated by [12],[13]:

$$
\begin{aligned}
P_{M_{D}} & =\left\lfloor 1-\left(1-P_{2}\right)^{M_{D}}\right\rfloor \\
& =1-\left(1-M_{D} P_{2}+\frac{M_{D}\left(M_{D}-1\right) P_{2}^{2}}{2 !}-\cdots+P_{2}^{M_{D}}\right)(5) \\
& \approx M_{D} P_{2} \quad \text { iff } \quad M_{D} \geq 4 \text { and } P_{2}<<1 .
\end{aligned}
$$

where $P_{2}$ denotes the pair-wise symbol error between adjacent (nearest-neighbour) symbols at distance $d$, given by:

$$
P_{2}=Q\left[\sqrt{\frac{d^{2}}{2 N_{0}}}\right]
$$

with $d$ defined in (4). In (6), $N_{0}$ denotes the one-sided noise power spectral density, and $\mathrm{Q}$ the well known Gauss tail probability, defined as $Q(x)=\frac{1}{\sqrt{2 \pi}} \int_{x}^{\infty} e^{-\frac{y^{2}}{2}} d y$. Now, when $M_{D} \geq 4$ and $P_{2}<<1$, equation (5), with a high degree of accuracy, simplifies to

$$
\begin{aligned}
P_{M_{D}} \approx M_{D} P_{2} & =M_{D} Q\left[\sqrt{\frac{4 E_{s}}{2 M_{D} N_{0}}}\right] \\
& =M_{D} Q\left[\sqrt{\frac{2 E_{b}}{N_{0}}}\right]
\end{aligned}
$$

By virtue of the Gray bit-encoding of nearest-neighbour symbols, the bit error rate (BER) in AWGN is well approximated by

$$
P_{b} \approx \frac{1}{M_{D}} P_{M_{D}}=P_{2}=Q\left[\sqrt{\frac{2 E_{b}}{N_{0}}}\right]
$$

Note that (8) is independent of the number of dimensions $M_{D}$. This implies that the BER of the proposed new QS-MDSO-WCDMA modulation scheme remains QPSK-like as $M_{D} \rightarrow \infty$, the only requirement being that a set of superorthogonal CSS be employed, such as the family presented in [8], or the extended-orthogonal (EO) sequences proposed in [14]. The primary implication of this is that the overall data throughput rate, and thus, the spectral efficiency (given a fixed spreading bandwidth), may be doubled (compared to QPSK) for every doubling of the number of dimensions, $M_{D}=4$. Moreover, the latter may be achieved by simply adding basic $M_{D}=4$-dimensional SO-WCDMA building blocks in parallel, using one new distinct CSS for each new set of $M_{D}=4$ dimensions, the only limitation being the available family size $M_{F}$ (i.e., the number of available CSS in a given set of SOCSS). The resulting composite multi-code (MC) modulator is called a MC-MD-SO-WCDMA modem. Consequently, upwards-expandability of the basic generic 4D-SO-WCDMA modulator may be achieved, while maintaining QPSK-like BER performance in AWGN as the data throughput linearly increases (doubles) with the addition of each new set of $M_{D}=4$ dimensions and one additional new CSS from the family of GCL-SO-CSS for each quadruple increase of dimensions.

Equation (8) is verified and confirmed in Fig. 3, depicting the BER performance for $M_{D}=4$ to $M_{D}=20$-dimensional QSMD-SO-WCDMA modulators in AWGN (the latter comprises $5 M_{D}=4$-dimensional building blocks in parallel). Note, for example, that the data throughputs of the $M_{D}=4$ to $M_{D}=16 \mathrm{QS}$ MD-SO-WCDMA modulation schemes correspond to the $M_{D}=2$-dimensional $M=2^{K=4}=16$ to $M=2^{K=16} \mathrm{QAM}$ modulated single carrier WCDMA modulators, i.e., 2 to 8 times that of QPSK, given identical spreading sequence lengths and bandwidths, where $M$ and $K$ are defined in (2). However, this is achieved by QS-MD-SO-WCDMA while maintaining the BER performance of QPSK, signifying a huge performance advantage over $M$-QAM single carrier and multi-carrier WCDMA modulation schemes when $M \geq 16$ (or $\left.M_{L} \geq \sqrt{M}=4\right)$.

A performance comparison between QS-MD-SO-WCDMA and $M$-QAM modulated WCDMA modulation schemes is presented in Table 1. The table lists various MD-SO-WCDMA schemes, together with an equivalent $M$-QAM WCDMA modulation scheme with identical normalized (with the spreading factor $L$ ) spectral efficiencies, $\eta_{f} L[\mathrm{~b} / \mathrm{s} / \mathrm{Hz}]$, where $L$ is identical for all modulation schemes considered.

\section{B. BER performance analysis in a Nakagami-fading multipath channel}

It can be shown that the theoretical BER for uncoded QS4D-SO-WCDMA (without CPM) in a Rayleigh fading multipath channel with $L_{m}$ independent paths, with or without an adaptive RAKE receiver with $L_{r}=L_{m}$ active taps, is given by [7],[18]:

$$
\begin{aligned}
P_{b} \approx & \frac{2}{\log _{2} M} \sqrt{\frac{\gamma_{s}}{1+\gamma_{s}}} \frac{\left(1+\gamma_{s}\right)^{-e_{s}} \Gamma\left(e_{s}+\frac{1}{2}\right)}{\sqrt{\pi}\left(e_{s}+1\right)} \\
& \times{ }_{2} F_{1}\left\{1, e_{s}+\frac{1}{2} ; e_{s}+1 ; \frac{1}{1+\gamma_{s}}\right\}
\end{aligned}
$$


where $\gamma_{s}=\left(\left(\gamma_{m} \Omega_{s}\right) / 2 e_{s}\right), \gamma_{m}=\gamma \sin ^{2}(\pi / M), \gamma=\frac{E \Omega_{0}}{N_{0}}$ and $E=P L T_{c}$, with $P$ the symbol power, $L$ the spreading sequence length, and $T_{c}$ the chip period. In (9) the natural Gray mapping of bits onto adjacent symbols in the QS-4D-SOWCDMA signal constellation has been used to obtain an approximation of the BER, $P_{b}$, given the expression of the symbol error rate, $P_{M}$. The function ${ }_{2} F_{1}\{a, b ; c ; z\}$ denotes the confluent hyper-geometric function and $\Gamma(\cdot)$ the Gamma function. $\Omega_{l}^{(k)}=\Omega_{0}^{(k)} e^{-\delta l}$ is the second moment of $\rho_{l}^{(k)}$, where $\Omega_{0}^{(k)}$ represents the second moment of the initial path strength of the $k^{\text {th }}$ user. The variable $\delta \geq 0$ denotes the rate of exponential decay of the multipath intensity profile of the channel. The $\left\{\rho_{l}^{(k)}\right\}$ are independent identically distributed (iid) Nakagami random variables. Also, $e_{s}=p \frac{q^{2}\left(L_{r}, \delta\right)}{q\left(L_{r}, 2 \delta\right)}$, and $\Omega_{s}=q\left(L_{r}, \delta\right)$, with $q(a, b)=\left(1-e^{-a b}\right) /\left(1-e^{-b}\right)$. The variable $p$ is a positive real number, such that $1 / p$ gives an indication of the "fading figure" of the channel [16].

Fig. 4 depicts the uncoded performance of the said QS-MDSO-WCDMA modulators with $M_{D}=4$ to $M_{D}=20$ in an exponentially-weighted three-path channel consisting of an initial Rician fading path, followed by two smaller exponentially weighted independently Rayleigh fading paths, without a complex RAKE processor in the receiver. The results are obtained for $k=1$ to 5 (maximum 7) simultaneous 4D users (i.e., the equivalent of a maximum of 28 BPSK users), with spreading sequences allocated from families of SO-CE-GCLCSS [7] of length $L=63$. Details of the experimental setup, and particularly the fading multipath channel, may be found in [18]. The BER-graphs for the AWGN and flat Rayleigh fading channels are also depicted, representing lower and upper performance bounds, respectively. Using the MUI-free family of CSS, results in an approximate 13 to $14 \mathrm{~dB}$ performance advantage over a single-user flat fading system, and a performance loss of only approximately $3.5 \mathrm{~dB}$ compared to the single-user AWGN bound at a BER of $P_{b}=10^{-3}$, signifying a virtually MUI-free receiver. Also note that there is a small deterioration in BER with an increase in the number of users for average signal-to-noise ratio per bit values exceeding $E_{b} / N_{o}=10 \mathrm{~dB}$, i.e., for BER values below $10^{-3}$. This asymptotic error rate plateau phenomenon may be attributed to the presence of multipath and the absence of a RAKE multipath combining processor in the receiver, as is verified by the results presented in Fig. 5.

Fig. 5 repeats the foregoing analysis on RUF families of CEGCL Zadoff-Chu (ZC) [15] and SO-CE-GCL-CSS [8] spreading sequences, respectively, with the presence of a complex $L_{r}=3$-finger RAKE processor in the receiver [18]. The theoretical BER graph obtained from (9) is also included for references purposes. The performance degradation of the non-ideal (i.e., not perfectly orthogonal) system employing ZC CE-GCL-CSS [15], compared to the essentially MUI-free performance of the modem with multiple users using spreading sequences from the family of SO-CE-GCL-CSS, is immediately evident. This degradation is attributed to the cross-correlation interference between users employing CSS from a family of non-super-orthogonal RUF Zadoff-Chu CECSS of length $L=63$, causing substantial MUI. Compared to the corresponding results in Fig. 4, it is noted that the incorporation of a complex RAKE processor has clearly reduced the BER degradation (spread) as a result of an increase in the number of users, as well as at least partly eradicated some of the asymptotic BER error rate plateau phenomenon observed in Fig. 4 for $E_{b} / N_{o}$ beyond $10 \mathrm{~dB}$ and $P_{b}$ below $10^{-3}$. The latter graph corresponds very well with the theoretical result defined in (9), the simulated results being within $1 \mathrm{~dB}$ of theory [7],[17],[18]. The use of SO-CE-GCLCSS therefore practically obviates the need for sophisticated MUI-cancellation techniques in the proposed new 4D-SOWCDMA modem, yielding a significantly simplified receiver and a suitable platform on which notable performance gains can be achieved through the incorporation of appropriate forward error (FEC) correction techniques.

\section{CONCLUSION}

A novel high performance power and spectrally efficient single carrier QS-4D-SO-WCDMA modem building block has been presented as an alternative to $M$-QAM single carrier and multi-carrier modulated OFDM and CDMA modulation standards employed in $3 \mathrm{G}$ or to be employed in next generation $(4 \mathrm{G})$ wireless access systems. It has been shown in [16] that the proposed QS-MD-SO-WCDMA modem can operate close to the $1 \mathrm{~dB}$ PA saturation point, with typically 3 to $6 \mathrm{~dB}$ less PA back-off than existing $M$-QAM modulated $M_{c}$-OFDM and $M_{c}$-CDMA modulation schemes. This signifies a notable communication range advantage, or alternatively, significantly improved handset battery life for a given performance level and range compared to existing methods. Notable MUI-free BER performance advantages have been demonstrated using a family of SO-CE-GCL-CSS [8], compared to conventional (Zadoff-Chu) CE-GCL-CSS [15], in both AWGN (Fig. 3) and multipath fading channels (Figs. 4 and 5), with and without RAKE maximum ratio combining (MRC). The MUI-free advantages are achieved with a lowcomplexity matched filter correlation-type receiver, without the need for complex MUI-mechanisms, but with the possible inclusion of RAKE MRC processing to exploit the presence of inherent multipath diversity, as well as appropriate FEC techniques to yield performance gains approaching the singleuser and even the Shannon bound.

The combination of the proposed new MD-WCDMA architecture with families of SO-CE-GCL-CSS renders a unique combination of spectral occupancy (i.e., minimum Nyquist bandwidth) and exceptional power efficiency by virtue of the near constant envelope (i.e., near constant instantaneous power) output signal, with data throughputs increasing with the addition of every basic 4D-WCDMA building block at the rate of $N_{D}$, where $N_{D}$ denotes the number of 4D modulators in parallel, while simultaneously maintaining the BER performance of BPSK/QPSK in both AWGN and Rayleigh fading multipath channels. The virtues 
of the proposed new MD-SO-WCDMA modulation schemes thus make it an attractive alternative for next generation wireless access and WLAN applications.

\section{REFERENCES}

[1] B. J. Choi, E. L. Kuan and L. Hanzo, "Crest-factor study of MC-CDMA and OFDM", In IEEE Vehicular Technology Conference (VTC'99), pp 233-237, 1999

[2] J.A. Davis and J. Jedwab, "Peak-to-mean power control in OFDM, Golay complementary sequences and Reed-Muller codes", IEEE Trans. on IT, vol. 45, no. 7, pp 2397- 2417, July 1999.

[3] C. Rössing and V. Tarokh, "A construction of OFDM 16-QAM sequences having low peak powers", IEEE Transactions on Information Theory, vol. 47, no. 5, pp 2091-2097, 2001.

[4] L.P. Linde and F.E. Marx, Multi-Dimensional Spread Spectrum Modem, United States complete patent no. 6744807, 1 June 2004.

[5] M. P. Lötter and L. P. Linde, "Constant envelope filtering of complex spreading sequences", IEE Electronics Letters, vol. 31, pp. 1406-1407, Aug. 1995.

[6] B. M. Popović, "Generalized Chirp-like polyphase sequences with optimum correlation characteristics", IEEE Trans. on IT, vol. IT-38, no. 4, pp 1404-1409, July 1992.

[7] S. I. Park, S. R. Park, I. Song and N. Suehiro, "Multiple-access interference reduction for QS-CDMA systems with a novel class of polyphase sequences", IEEE Trans. on Information Theory, vol. 46, no. 4, pp 1448-1458, July 2000.

[8] L. P. Linde, I. Pryra and S. A. Swanepoel, "On a new family of complex spreading sequences with zero cross-correlation properties", The Transactions of the South African Institute of Electrical Engineers (SAIEE), vol. 94, pp 50-56, Dec. 2003.

[9] L. P. Linde and J. D. Vlok, "Power and spectrally efficient fourdimensional super-orthogonal WCDMA building block for next generation wireless applications", IEEE Communications Letters, pp. 519-521, July 2006.

[10] A. Scaglione, G. B. Giannakis and S. Barbarossa, "Lagrange/ Vandermonde MUI eliminating user codes for quasi-synchronous CDMA in unknown multipath", IEEE Trans. on Signal Processing, vol. 48, no. 7, pp 2057-2073, 2000.

[11] F. E. Marx, DSSS communication link employing complex spreading sequences, M.Eng dissertation, University of Pretoria, Pretoria, South Africa, Aug. 2005.

[12] J. M. Wozencraft and I. M. Jacobs, Principles of Communication Engineering, Chapter 4 pp 254-257, Wiley, New York, 1965.
[13] L. Luand and V. K. Dubey, "Extended orthogonal polyphase codes for multicarrier CDMA systems", IEEE Comm. Letters, vol. 8, no. 12, pp 700-702, Dec. 2004.

[14] D. C. Chu, "Polyphase codes with good periodic correlation properties", IEEE Trans on IT, vol. 18, no. 4, pp 531-532, April 1972.

[15] L. P. Linde and F. E. Marx, "Power and spectral efficiency performance of a family of WCDMA-modulated constant-envelope root-of-unity filtered complex spreading sequences in non-linear power amplification", The Transactions of the SAIEE, vol. 94, no. 4, pp 57-67, Dec. 2003.

[16] T. Eng and L. B. Milstein, "Coherent DS-CDMA performance in Nakagami multipath fading", IEEE Trans. on Commun., vol. 43, no. 2/3/4, pp 1134-1143, Feb/Mar/April 1995.

[17] L. Staphorst, Viterbi Decoded Linear Block Codes for Narrowband and Wideband Communication over Mobile Fading Channels, pp 95-98. M.Eng dissertation, University of Pretoria, Pretoria, South Africa, July 2005.

Louis P. Linde (M'80-SM'90) obtained the Hons. B. Eng (ElectroTechnical) degree from the University of Stellenbosch, South Africa in 1973, and the M.Eng (Electronic) (cum laude) and the D.Eng degrees from the University of Pretoria, Pretoria, South Africa in 1980 and 1983, respectively. $\mathrm{He}$ is currently Professor and Group Head of the Signal Processing and Telecommunications division in the Department of Electrical, Electronic and Computer Engineering at the University of Pretoria, Pretoria, South Africa, where he is also Director of the research Centre for Radio and Digital Communication (CRDC). His research interests are in the fields of digital modulation and coding for fading channels, CDMA spreading sequence design, synchronization and equalization methods for highly mobile communication applications and MIMO space-time coding.

Prof. Linde is the specialist editor, Telecommunications, Signal Processing and Information Theory, of the SAIEE Africa Research Journal.

Jacobus D. Vlok obtained the B. Eng (Electronic), the B. Eng (Honns) and M. Eng degrees (all cum laude) from the University of Pretoria, South Africa, in 2004, 2005 and 2007, respectively. The title of his Master's dissertation (cum laude) was: Sparse Graph Codes on a Multidimensional WCDMA Platform. His specialist research field is coding for the fading channel, with the emphasis on trellis decoding of block codes, as well as Turbo, LDPC and Repeat-Accumulate coding techniques.

Table 1. Performance comparison of MD-SO-WCDMA and $M$-QAM WCDMA modulation schemes in AWGN. To facilitate direct comparison, equal spreading sequence length $L$ and spreading bandwidths $B_{s}$ are assumed in each case.

\begin{tabular}{|c|c|c|c|c|c|c|}
\hline Modulation Scheme & $\begin{array}{c}\text { Number of } \\
\text { Dimensions } \\
M_{D}\end{array}$ & $\begin{array}{c}\text { Number of } \\
\text { levels per } \\
\text { dimension } \\
M_{L}\end{array}$ & $\begin{array}{c}\begin{array}{c}\text { Number of } \\
\text { symbols }\end{array} \\
M=\left(M_{L}\right)^{M_{D}}\end{array}$ & $\begin{array}{c}\begin{array}{c}\text { Spectral } \\
\text { efficiency }\end{array} \\
\eta_{f} \cdot L \\
{[\mathrm{~b} / \mathbf{s} / \mathbf{H z}]}\end{array}$ & $\begin{array}{c}E_{b} / N_{0} @ \\
P_{b}=10^{-6} \\
\\
{[\mathrm{~dB}]}\end{array}$ & $\begin{array}{c}\text { MD-SO- } \\
\text { WCDMA } \\
\text { SNR Gain } \\
\text { [dB] }\end{array}$ \\
\hline 4D-SO-WCDMA & 4 & 2 & 16 & 4 & 10.53 & 4.15 \\
\hline 16-QAM-WCDMA & 2 & 4 & $"$ & " & 14.68 & \\
\hline 8D-SO-WCDMA & 8 & 2 & 256 & 8 & 10.53 & 13.5 \\
\hline 256-QAM-WCDMA & 2 & 16 & $"$ & " & 24.03 & \\
\hline 12D-SO-WCDMA & 12 & 2 & $2^{12}$ & 12 & 10.53 & 23.81 \\
\hline $2^{12}$-QAM-WCDMA & 2 & $2^{6}$ & $"$ & $"$ & 34.34 & \\
\hline
\end{tabular}




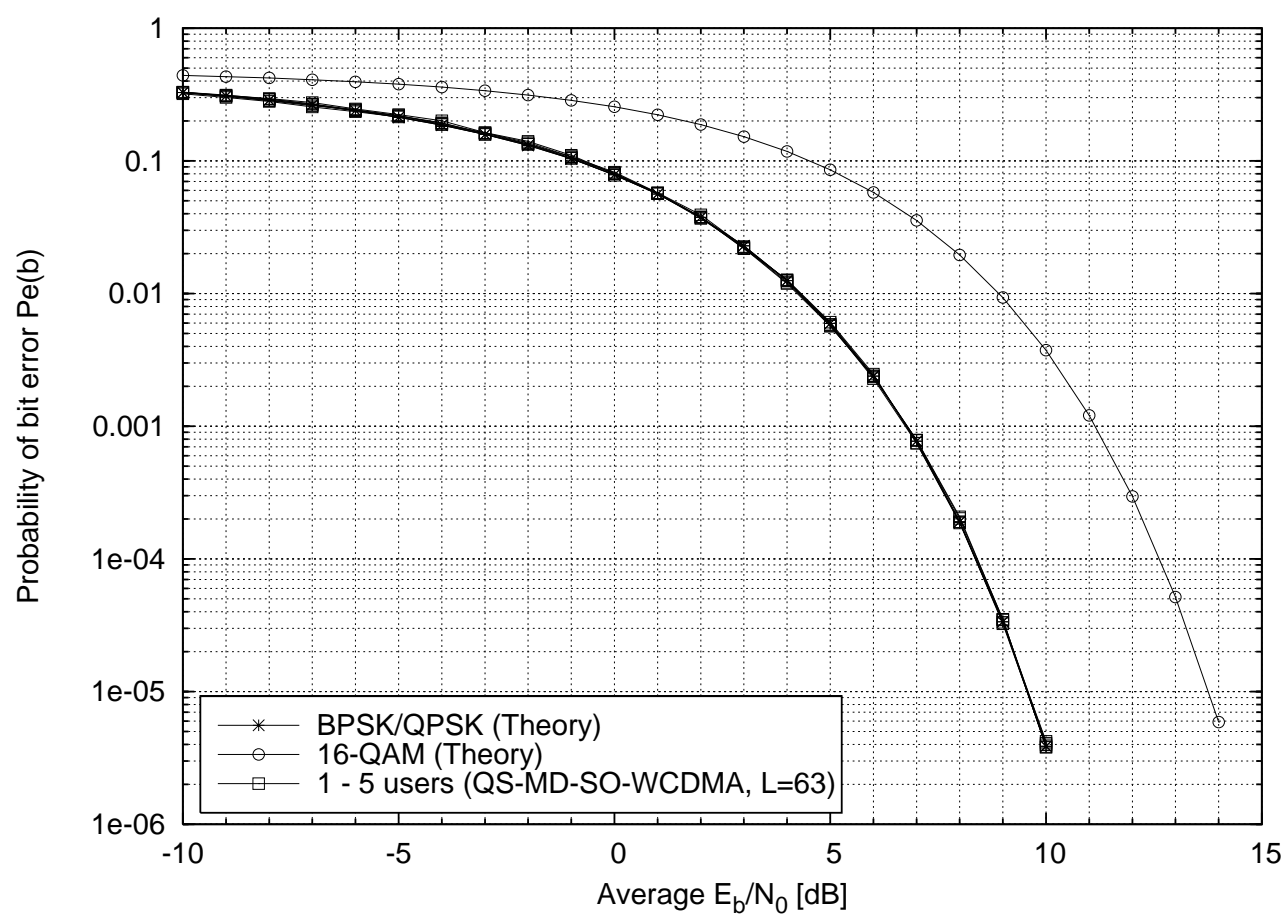

Fig. 3 Simulated uncoded BER performance graphs in AWGN for 4D- to 20D-SO-WCDMA, compared to 2D-QPSK WCDMA and 16-QAM WCDMA. The former MD-SO-CDMA systems facilitate data throughputs of 2 to 10 times 2D-QPSK WCDMA, and 1 to 5 times 16-QAM WCDMA, respectively, given identical spreading sequence lengths $\mathrm{L}$ and Nyquist filtered $(\alpha=0)$ spreading bandwidths, $\mathrm{B}_{\mathrm{s}}$.

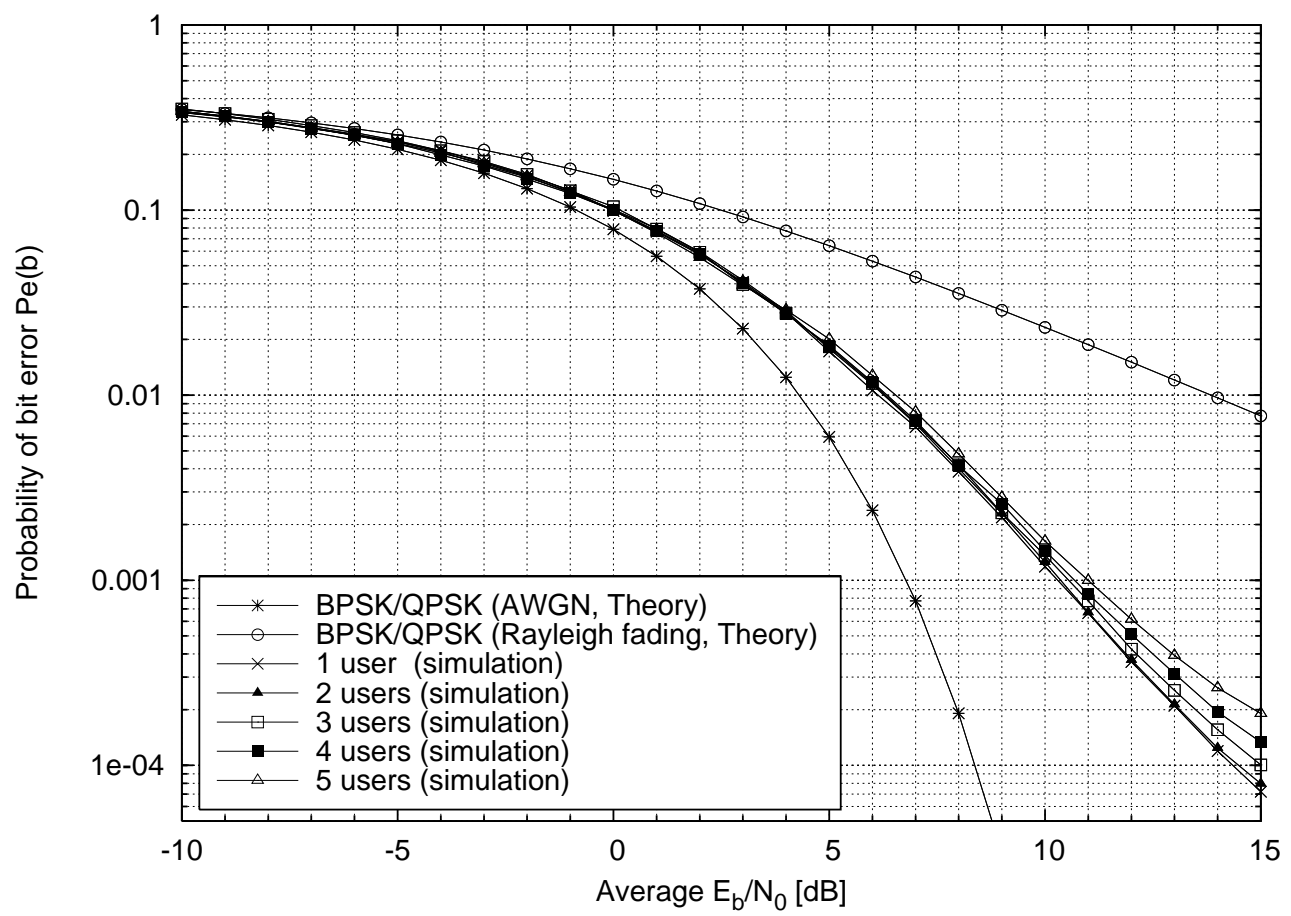

Fig. 4 Uncoded multi-user BER performance of QS-4D-SO-WCDMA employing SO-CE-GCL-CSS of length $L=63$ in an exponentially weighted Multipath Rayleigh fading channel with an initial Ricean and two statistically independent Rayleigh fading multipaths (see [17] pp 95-98 for details). Results for a maximum of 5 basic QS-4D-SO-WCDMA systems are shown, which is equivalent to 20 conventional BPSK users in parallel. 


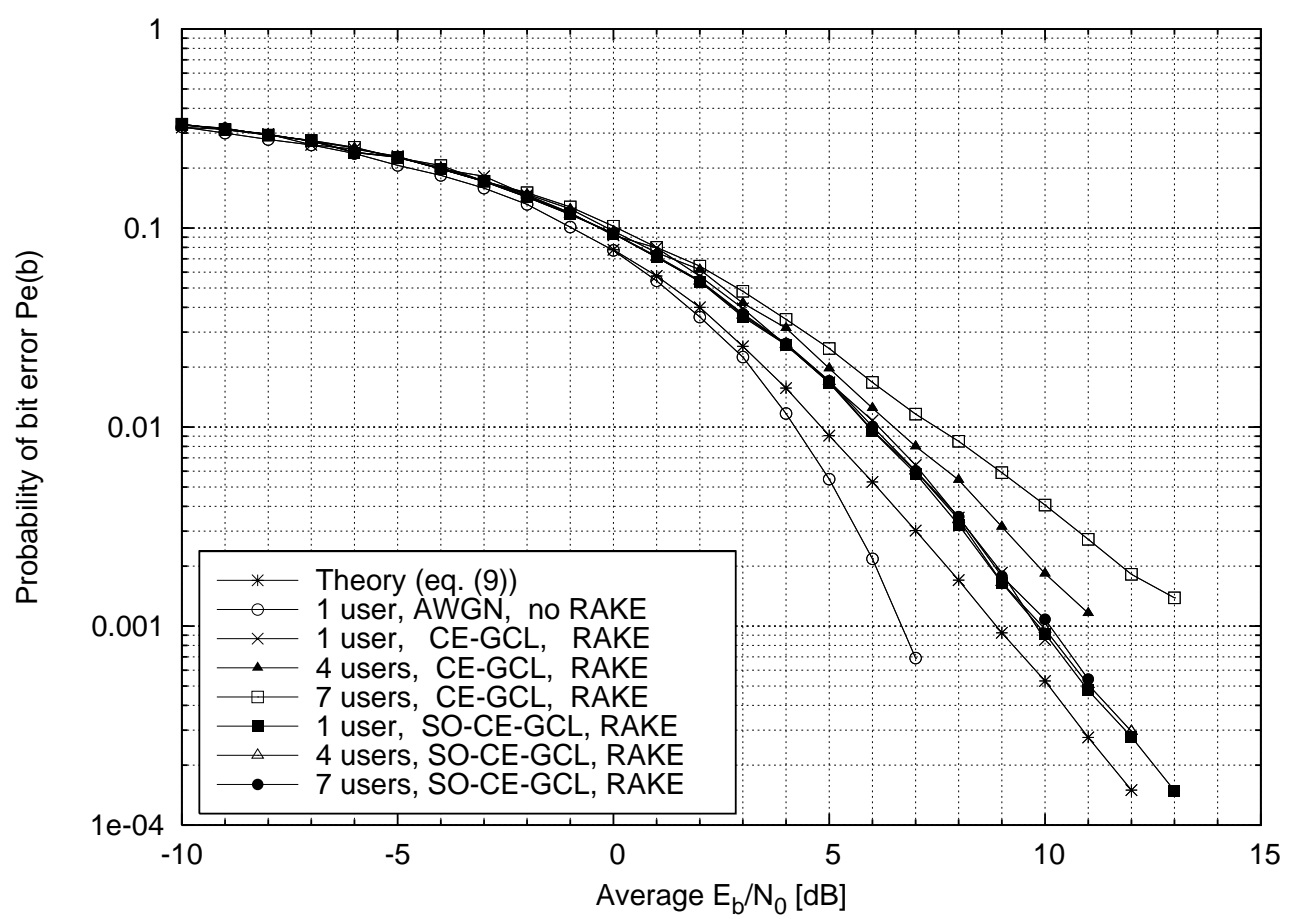

Fig. 5 Multi-user BER performance of QS-4D-SO-WCDMA SO-CE-GCL-CSS versus CE-GCL (Zadoff$\mathrm{Chu}$ ) sequences of length $L=63$ in a Multipath Rayleigh fading channel with $L_{m}=3$ paths (one Rician and two statistically independently Rayleigh fading), with a complex RAKE receiver processor with $L_{r}=3$ active fingers. The single user AWGN bound (without RAKE) and the theoretical result of equation (9) are included for reference purposes. 